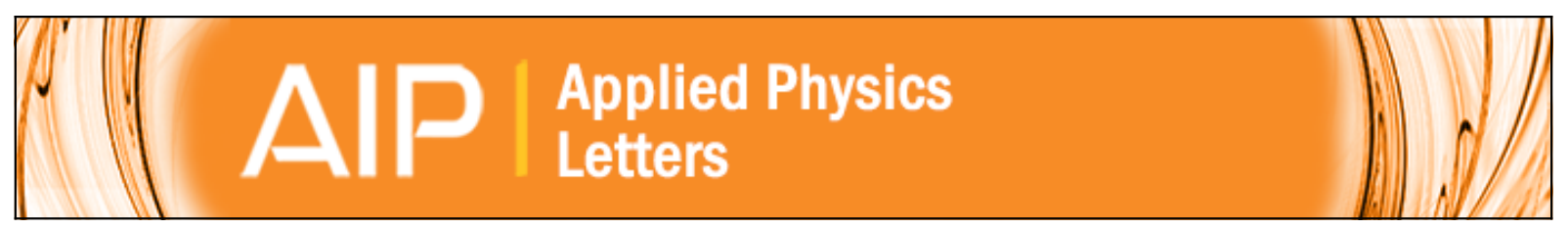

\title{
Direct writing of birefringent elements by ultrafast laser nanostructuring in multicomponent glass
}

S. S. Fedotov, R. Drevinskas, S. V. Lotarev, A. S. Lipatiev, M. Beresna, A. Čerkauskaitè, V. N. Sigaev, and P. G. Kazansky

Citation: Applied Physics Letters 108, 071905 (2016); doi: 10.1063/1.4941427

View online: http://dx.doi.org/10.1063/1.4941427

View Table of Contents: http://scitation.aip.org/content/aip/journal/apl/108/7?ver=pdfcov

Published by the AIP Publishing

\section{Articles you may be interested in}

Investigation on the near-infrared-emitting thermal stability of $\mathrm{Bi}$ activated alkaline-earth aluminoborosilicate glasses

J. Appl. Phys. 117, 053107 (2015); 10.1063/1.4907565

In-situ characterization of femtosecond laser-induced crystallization in borosilicate glass using time-resolved surface third-harmonic generation

Appl. Phys. Lett. 103, 201116 (2013); 10.1063/1.4831655

Femtosecond laser-induced refractive index modification in multicomponent glasses

J. Appl. Phys. 97, 083102 (2005); 10.1063/1.1876578

Femtosecond laser-pulse-induced birefringence in optically isotropic glass

J. Appl. Phys. 95, 5280 (2004); 10.1063/1.1707231

Three-photon-excited fluorescence of Al $2 \mathrm{O} 3$ - SiO 2 glass containing Eu 3+ ions by femtosecond laser irradiation

Appl. Phys. Lett. 84, 2076 (2004); 10.1063/1.1688984

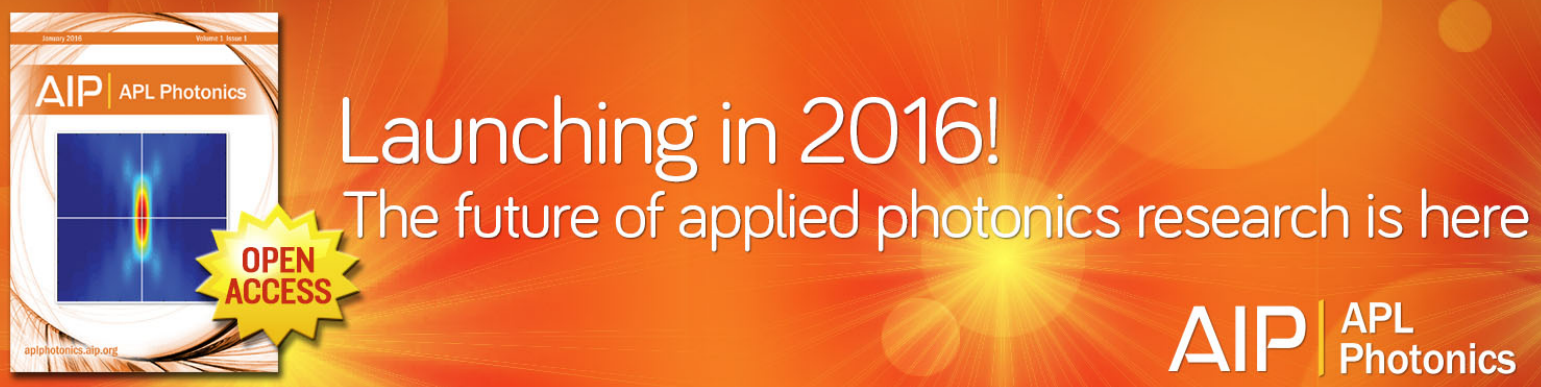




\title{
Direct writing of birefringent elements by ultrafast laser nanostructuring in multicomponent glass
}

\author{
S. S. Fedotov, ${ }^{1}$ R. Drevinskas, ${ }^{2}$ S. V. Lotarev,${ }^{1, a)}$ A. S. Lipatiev, ${ }^{1}$ M. Beresna, ${ }^{2}$ \\ A. Čerkauskaitè, ${ }^{2}$ V. N. Sigaev, ${ }^{1}$ and P. G. Kazansky ${ }^{1,2}$ \\ ${ }^{1}$ International Center of Laser Technology, D. Mendeleyev University of Chemical Technology of Russia, \\ Miusskaya Sq., Moscow 125047, Russia \\ ${ }^{2}$ Optoelectronics Research Centre, University of Southampton, Southampton SO17 1BJ, United Kingdom
}

(Received 24 November 2015; accepted 19 January 2016; published online 17 February 2016)

\begin{abstract}
Self-assembled nanostructures created by femtosecond laser irradiation are demonstrated in alkalifree aluminoborosilicate glass. The growth of the induced retardance associated with the nanograting formation is three orders of magnitude slower than in silica glass and is observed only within a narrow range of pulse energies. However, the strength of retardance asymptotically approaches the value typically measured in pure silica glass, which is attractive for practical applications. A similar intensity threshold for nanograting formation of about $1 \mathrm{TW} / \mathrm{cm}^{2}$ is observed for all glasses studied. The radially polarized vortex beam micro-converter designed as a space-variant quarter-wave retarder for the near-infrared spectral range is imprinted in commercial Schott AF32 glass. C 2016 AIP Publishing LLC. [http://dx.doi.org/10.1063/1.4941427]
\end{abstract}

More than a decade ago, a femtosecond laser-driven self-assembly process phenomenon was first observed in the bulk of silica glass. ${ }^{1,2}$ Nanostructures formed during this process, frequently referred to as nanogratings, exhibit a number of interesting properties including anisotropic light scattering, ${ }^{1}$ wavelength-dependent reflectivity, ${ }^{1}$ and form birefringence with a high resistance to temperature. ${ }^{3}$ Recently, these properties were successfully harnessed for multiple practical applications including spatially variant polarization optics, ${ }^{4}$ microfluidics, ${ }^{5}$ polarization selective holography, ${ }^{6}$ and ultrastable optical data storage. ${ }^{7,8}$

For a long time, the formation of nanogratings was thought to be only observed in a handful of materials. This contradicts the context of surface periodic nanostructures, known as surface ripples, which were observed virtually on any type of material ranging from amorphous ${ }^{9}$ and crystalline $\mathrm{e}^{10}$ dielectrics to semiconductors ${ }^{11}$ and metals. ${ }^{12}$ However, nanogratings were later observed in the bulk of crystalline materials such as $\mathrm{TeO}_{2}{ }^{13,14}$ and sapphire. ${ }^{15}$ Furthermore, doping of pure silica glass with Ge, F, and $\mathrm{P}$ was demonstrated to affect the threshold for nanograting formation. ${ }^{16}$ Recently, evidence for femtosecond laser-induced nanogratings has been observed in glasses other than $\mathrm{SiO}_{2}$, including several studies reported nanogratings in $\mathrm{GeO}_{2}$ glass, ${ }^{17-19}$ binary titanium silicate glass (ULE, Corning), ${ }^{20,21}$ and two multicomponent borosilicate glasses (BK7 and Borofloat 33, Schott). ${ }^{20,22}$

Germanium dioxide is formed by a three-dimensional network of $\left[\mathrm{GeO}_{4}\right]$ tetrahedra with all bridging oxygen atoms, whereas ULE glass also possesses structure similar to pure $\mathrm{SiO}_{2}$ in which $\mathrm{Ti}$ atoms substitute for $\mathrm{Si}$ in the same structural sites. Borofloat 33 is a multicomponent borosilicate glass $\left(81 \% \mathrm{SiO}_{2}, 13 \% \mathrm{~B}_{2} \mathrm{O}_{3}, 4 \% \mathrm{Na}_{2} \mathrm{O} / \mathrm{K}_{2} \mathrm{O}\right.$, and $2 \%$ $\mathrm{Al}_{2} \mathrm{O}_{3}{ }^{23}$ ) with a more complex chemical structure.

\footnotetext{
${ }^{\text {a) }}$ Author to whom correspondence should be addressed. Electronic mail: sergey_lot@mail.ru
}

Unexpectedly, laser-induced nanostructures observed in Borofloat 33 glass demonstrated an extremely fine period of $\sim 60 \mathrm{~nm}^{22}$ Nanostructures in BK7 glass $\left(70 \% \mathrm{SiO}_{2}, 11.5 \%\right.$ $\mathrm{B}_{2} \mathrm{O}_{3}, 9.5 \% \mathrm{Na}_{2} \mathrm{O}, 7.5 \% \mathrm{~K}_{2} \mathrm{O}, 1 \% \mathrm{BaO}$, and traces of $\mathrm{TiO}_{2}$ and $\mathrm{CaO})^{24}$ were not revealed using scanning electron microscopy despite the induced retardance was of the same level as in Borofloat $33 .{ }^{20}$ The birefringence in both multicomponent glasses could only be observed in a very limited range of pulse durations (150-200 fs) and the induced retardance was more than one order of magnitude weaker than in fused silica glass.

Here, we demonstrate that nanogratings can be formed in alkali-free aluminoborosilicate AF32 glass (Schott). Under optimized experimental conditions, the retardance reaches values in excess of $200 \mathrm{~nm}$ (an equivalent of quarterwave for $800 \mathrm{~nm}$ ) comparable to the values typical for silica glass. As a demonstration, we imprinted a micro-converter in the bulk of AF32 glass for generating a radially polarized optical vortex.

Samples selected for the ultrafast laser processing were cut from $1 \mathrm{~mm}$ thick plane-parallel plates of commercial AF32 glass (Schott) and polished to an optical grade. For comparison, we also used plates of commercially available UVSIL silica glass and Borofloat 33 glass (Schott). Modification of glass samples was carried out with an Yb:KGW-based regeneratively amplified femtosecond laser system (Pharos Light Conversion, Ltd.) operating at the wavelength of $1030 \mathrm{~nm}$ emitting pulses with a duration variable from 300 fs to $10 \mathrm{ps}$ at an adjustable repetition rate up to $500 \mathrm{kHz}$. Pulse energy was controlled by the combination of a half-wave plate and a Glan polarizer and measured after an objective focusing the beam into glass samples. Additional half-wave plate was used to control an orientation of linear polarization of the laser beam, which was focused inside the glass samples with 0.35 or 0.55 numerical aperture aspheric lenses.

Birefringence parameters of laser imprinted structures were analyzed with a quantitative birefringence measurement 
system (Abrio Microbirefringence, Cri, Inc.) integrated into an optical microscope (Olympus BX51). This system allows mapping of the slow axis orientation and measurement of the absolute value of the retardance. The structural analysis of glass samples was performed with a scanning electron microscope (TESCAN Vega 3 LMU) operating in a secondary electron imaging mode. The laser modified regions were exposed for the SEM analysis by polishing and subsequent etching in 0.5 wt. \% hydrofluoric acid for $7 \mathrm{~min}$.

A set of lines was inscribed by translating a sample perpendicularly to the laser propagation direction. In this experiment, an aspheric $0.35 \mathrm{NA}$ lens was used for focusing the laser beam $50 \mu \mathrm{m}$ below the surface. The pulse duration was varied from $300 \mathrm{fs}$ to $5 \mathrm{ps}$, and the repetition rate was set to $100 \mathrm{kHz}$. The optical characterization of the irradiated structures in AF32 glass revealed the presence of laser induced birefringence with a well-defined slow axis orientation. Similarly to silica glass, the slow axis in the imprinted structures was always perpendicular to the polarization of the writing laser beam.

The secondary electron image (Fig. 1(a)) of the area irradiated with $600 \mathrm{fs}, 150 \mathrm{~nJ}$ pulses at a $100 \mathrm{kHz}$ repetition rate with a 5000 pulse/ $\mu \mathrm{m}$ density using a $0.45 \mathrm{NA}$ objective revealed a nanograting with a period of about $100 \mathrm{~nm}$. Unexpectedly, this value is much smaller than the period reported for nanogratings induced in silica glass, which is typically above $200 \mathrm{~nm}$ at the irradiation wavelength of $1030 \mathrm{~nm} .{ }^{25}$ On the other hand, the obtained period is slightly larger than a $60 \mathrm{~nm}$ period reported for the nanogratings in Borofloat 33 glass..$^{20,22}$

Retardance dependence on the pulse duration and pulse energy was measured for AF32, Borofloat 33 and silica glasses for the same irradiation conditions (Figs. 1(b)-1(d)). Both AF32 and silica glasses exhibited a similar energy threshold above which birefringence could be observed. Dependence on the pulse duration indicates that the threshold depends on the
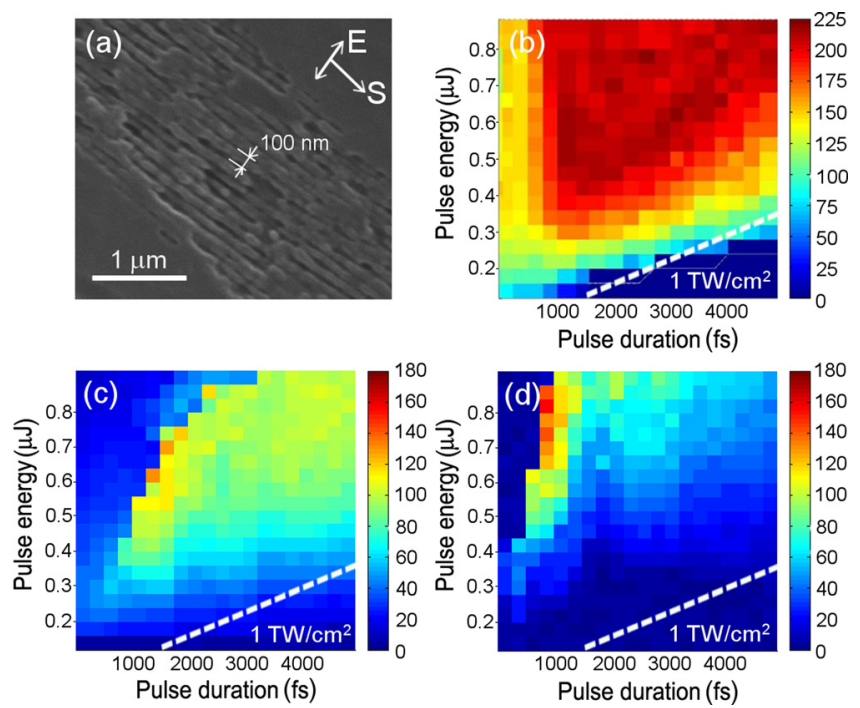

FIG. 1. Secondary electron image of AF32 glass surface after exposing and relieving of laser-imprinted structure ( $E$ is the orientation of electric field in the laser beam, and $S$ is the scanning direction) (a). Pseudocolor plots of retardance as a function of pulse energy and duration for silica (b), AF32 (c), and Borofloat 33 (d) glasses at $3 \mu \mathrm{m} / \mathrm{s}$ laser scanning speed and $100 \mathrm{kHz}$ pulse repetition rate, which is equivalent to nearly $8 \times 10^{4}$ pulses per dot. intensity and not on the amount of deposited energy, i.e., fluence. We estimated this threshold is about $1 \mathrm{TW} / \mathrm{cm}^{2}$ for these two glasses. In the case of AF32, the dependence of retardance on the pulse duration is more complicated, exhibiting a local maximum at $\sim 1-2 \mathrm{ps}$ and a minimum at $\sim 2.5 \mathrm{ps}$ followed by slow growth up to $5 \mathrm{ps}$. This trend is even more pronounced for Borofloat 33 glass which manifested comparatively strong and narrow retardance maximum shifted towards shorter pulse durations as compared to AF32 glass. With respect to pulse energy, both lower and upper thresholds for Borofloat 33 of birefringence are shifted upwards in comparison to AF32 glass (Fig. 1(d)). The maximum value of retardance induced with a certain pulse energy increases, and the retardance peak position shifts to longer pulse durations with increasing pulse energy. Interestingly, a previous study ${ }^{20}$ on nanogratings in Borofloat 33 glass revealed a similar dependence of retardance on pulse duration with maximum retardance measured at about 150-200 fs. Our observations obviously complement this result within different ranges of pulse energy and duration.

Additionally, we observed strong polarization dependence on the induced retardance. For all of the studied glasses, a beam polarized perpendicularly to the writing direction resulted in nearly $20 \%-25 \%$ higher values of retardance than in the case for parallel polarization in agreement with a previous study. ${ }^{26}$ To avoid this effect, we continued our experiments in static irradiation conditions, where a series of dots were written using a stationary beam focused with a $0.55 \mathrm{NA}$ aspheric lens $30 \mu \mathrm{m}$ below the glass surface. The first set of dots was written with fixed pulse energy of $21 \mathrm{~nJ}$ and a number of pulses increasing by a factor of 2 from dot to dot in the range from 4 to $262144\left(2^{18}\right)$. Analogous sets were written with a pulse energy being increased by a factor of 1.18 from set to set up to $213 \mathrm{~nJ}$. Retardance of the laser-imprinted birefringent structures in AF32, UVSIL, and Borofloat 33 glasses was measured as a function of the energy and number of pulses at a fixed pulse duration of $600 \mathrm{fs}$ and repetition rate of $200 \mathrm{kHz}$ (Fig. 2).

Unlike in silica glass where retardance is observed after 10-20 pulses for larger pulse energy (Fig. 2(c)), strong retardance in AF32 glass was induced after a significant number of pulses, typically larger than $3 \times 10^{4}$. However, weak, but measurable retardance could be detected after several hundred of pulses. Interestingly, with 600 fs laser pulses, the birefringence in AF32 could be induced only within a comparatively narrow range of pulse energies of about 40-170 $\mathrm{nJ}$ with the strongest retardance values at about $95 \mathrm{~nJ}$ pulse energy. For Borofloat 33 glass, weak retardance was measured throughout the whole range of energies and number of pulses used in the experiments (Fig. 2).

Similar trends were obtained for the different repetition rates $(25,100,300$, and $400 \mathrm{kHz})$ but absolute values of retardance varied to a certain degree depending on the selected rate (Fig. 3). Generally, the strongest retardance observed in AF32 glass was measured at repetition rates of 100 and $200 \mathrm{kHz}$ (Fig. 3, inset). Analyzing AF32 glass, we also observed two small peaks of retardance at about $2 \times 10^{2}$ and $8 \times 10^{3}$ pulses for 100,200 , and $300 \mathrm{kHz}$. Borofloat 33 glass also showed some dependence of retardance on the pulse repetition rate, but the signal-to-noise ratio was too small to draw any conclusions (Fig. 3, inset). 

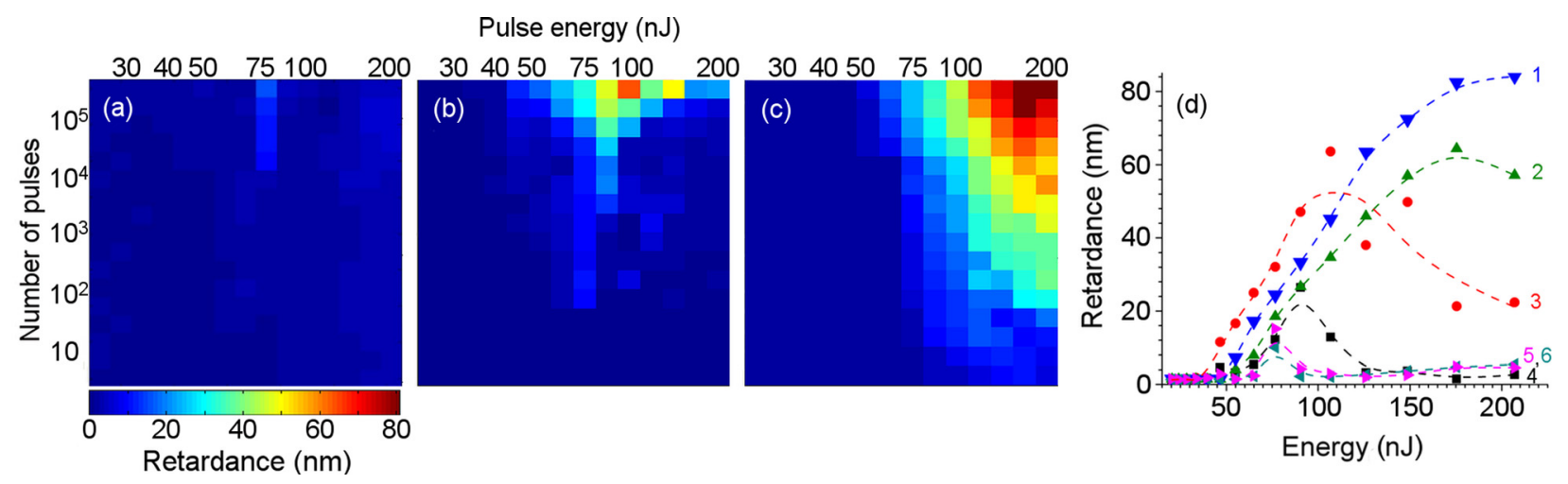

FIG. 2. Pseudocolor plots of retardance induced by the stationary beam as a function of pulse energy and number of pulses for Borofloat 33 (a), AF32 (b), and silica (c) glasses at $600 \mathrm{fs}$ pulse duration and $200 \mathrm{kHz}$ pulse repetition rate; plots of retardance versus pulse energy (d) for silica (1/ $\mathbf{\nabla}-262144\left(2^{18}\right)$

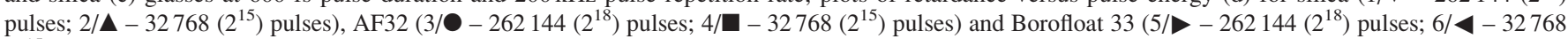
$\left(2^{15}\right)$ pulses) glasses.

The experiments reveal that unlike silica glass, nanogratings with significant retardance in multicomponent glasses can only be obtained within comparatively narrow ranges of pulse energy and pulse duration. In each particular case, there is a specific range of optimal pulse parameters, which appears to be narrower for Borofloat 33 than for AF32. General trends for these two glasses are: (a) a pronounced intensity threshold below which nanogratings cannot be induced; (b) much higher number of pulses, which is about $10^{4}-10^{5}$, is required to obtain a noticeable retardance as compared to silica glass. In the context of our experimental results, AF32 glass seems being an intermediate case between Borofloat 33 and silica glasses in terms of the nanograting formation.

Comparing the chemical composition of these glasses can provide some useful information for understanding this difference. The chemical composition of $\mathrm{AF} 32$ glass is in the range of $55 \%-65 \% \mathrm{SiO}_{2}, 15 \%-20 \% \mathrm{Al}_{2} \mathrm{O}_{3}, 5 \%-10 \% \mathrm{~B}_{2} \mathrm{O}_{3}$, $10 \%-15 \%$ alkaline earth oxides, whereas Borofloat 33 consists of $81 \% \quad \mathrm{SiO}_{2}, 13 \% \quad \mathrm{~B}_{2} \mathrm{O}_{3}, 4 \% \quad \mathrm{Na}_{2} \mathrm{O} / \mathrm{K}_{2} \mathrm{O}$, and $2 \%$ $\mathrm{Al}_{2} \mathrm{O}_{3}{ }^{22}$ Glass transition temperatures are $525^{\circ} \mathrm{C}, 717^{\circ} \mathrm{C}$, and $\sim 1250^{\circ} \mathrm{C}$ for Borofloat $33, \mathrm{AF} 32$, and silica glass, respectively. Despite, AF32 contains much less silica oxide than Borofloat 33, an addition of $\mathrm{Al}_{2} \mathrm{O}_{3}$ provides a fairly more

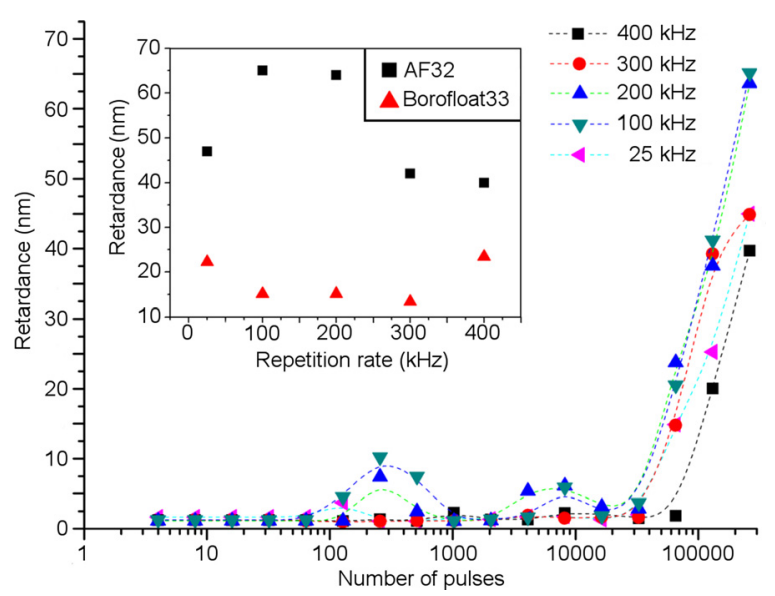

FIG. 3. Retardance in AF32 glass induced by the stationary beam vs. number of pulses at different pulse repetition rates (pulse energy $107 \mathrm{~nJ}$; pulse duration $600 \mathrm{fs}$ ); maximal retardance obtained vs. pulse repetition rate for AF32 and Borofloat 33 glasses is shown in the inset. rigid structure increasing its transition temperature. Thus, the presence of alkaline cations rather than amount of silica oxide seems to have a more significant effect on nanograting formation. Moreover, high pulse repetition rates can lead to a diffusion of alkaline and alkaline earth cations out of the center of the irradiation zone in silicate glasses as was demonstrated by examples of $\mathrm{Na}$ (Ref. 27) and $\mathrm{Ca}$ (Ref. 28). Large number of pulses required to induce nanogratings in the studied glasses might indicate that slow diffusion of alkali ions cations could precede the appearance of a nanograting.

The large induced retardance obtained in AF32 glass can be exploited for the benefit of practical applications. As a demonstration, a space variant quarter-wave plate operating in the near-infrared for the generation of a radially polarized optical vortex beam was imprinted in the bulk of AF32 glass (Fig. 4). ${ }^{4}$ The optical element was inscribed with a $0.16 \mathrm{NA}$ objective at $1 \mu \mathrm{m}$ interline distance using $0.75 \mu \mathrm{J}$ $600 \mathrm{fs}$ laser pulses at a $200 \mathrm{kHz}$ repetition rate. The scanning speed was set to $1 \mu \mathrm{m} / \mathrm{s}$.

In conclusion, we observed ultrafast laser induced selfassembled nanostructures in commercial alkali-free aluminoborosilicate AF32 glass (Schott). Analyzing from birefringence exhibited by nanostructures formed in AF32, silica, and borosilicate Borofloat 33 (Schott) glasses, we observed that stronger measured retardance correlates with higher glass transition temperature rather than with higher content of $\mathrm{SiO}_{2}$ in glasses. We also observed that for all glasses, there is a similar intensity threshold of about $1 \mathrm{TW} / \mathrm{cm}^{2}$ for

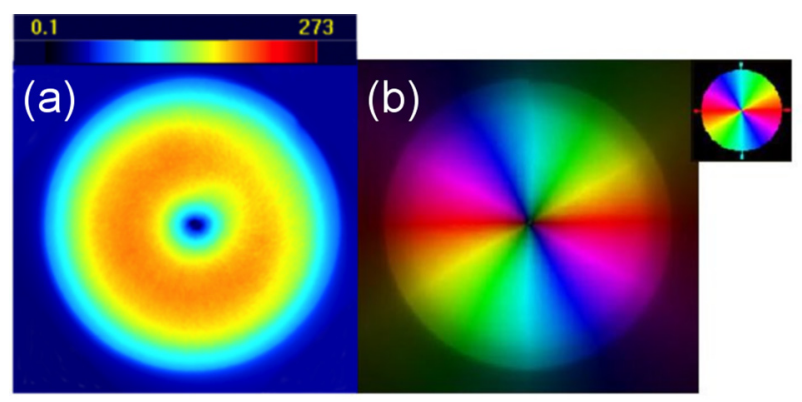

FIG. 4. Quantitative birefringence measurement of $100 \mu \mathrm{m}$ diameter polarization converter imprinted in the bulk of AF32 glass: (a) retardance (nm), and (b) orientation of the slow axis, with its corresponding pseudo colors (insets). 
nanograting formation. AF32 manifests measurable laserinduced birefringence in a comparatively narrow pulse energy range after extended laser irradiation. However, retardance in AF32 glass obtained at optimal irradiation conditions is of the same order of magnitude as observed in silica glass and can be exploited for practical applications such as polarization sensitive optical elements.

This study was financially supported by the Ministry of Education and Science of the Russian Federation (Grant No. 14.Z50.31.0009) and the Engineering and Physical Sciences Research Council (EPSRC Project No. EP/M029042/1). The authors would like to thank Stefan Hermes from Schott AG for supplying the AF32 glass samples and I. C. Avetissov from D, Mendeleyev University of Chemical Technology of Russia for performing SEM. The data for this work are accessible through the University of Southampton Institutional Research Repository (http://dx.doi.org/10.5258/ SOTON/385405).

${ }^{1}$ P. G. Kazansky, H. Inouye, T. Mitsuyu, K. Miura, J. Qiu, K. Hirao, and F. Starrost, Phys. Rev. Lett. 82, 2199 (1999).

${ }^{2}$ Y. Shimotsuma, P. G. Kazansky, J. R. Qiu, and K. Hirao, Phys. Rev. Lett. 91, 247405 (2003).

${ }^{3}$ E. Bricchi and P. G. Kazansky, Appl. Phys. Lett. 88, 111119 (2006).

${ }^{4}$ M. Beresna, M. Gecevičius, P. G. Kazansky, and T. Gertus, Appl. Phys. Lett. 98, 201101 (2011).

${ }^{5}$ Y. Bellouard, A. Said, M. Dugan, and P. Bado, Opt. Express 12, 2120 (2004).

${ }^{6}$ W. Cai, A. R. Libertun, and R. Piestun, Opt. Express 14, 3785 (2006).

${ }^{7}$ Y. Shimotsuma, M. Sakakura, P. G. Kazansky, M. Beresna, J. Qiu, K. Miura, and K. Hirao, Adv. Mater. 22, 4039 (2010).

${ }^{8}$ J. Zhang, M. Gecevičius, M. Beresna, and P. G. Kazansky, Phys. Rev. Lett. 112, 033901 (2014).
${ }^{9}$ A. P. Joglekar, H. Liu, G. J. Spooner, E. Meyhöfer, G. Mourou, and A. J. Hunt, Appl. Phys. B 77, 25 (2003).

${ }^{10}$ A. M. Ozkan and A. P. Maishe, Appl. Phys. Lett. 75, 3716 (1999).

${ }^{11}$ A. Borowiec and H. K. Haugen, Appl. Phys. Lett. 82, 4462 (2003).

${ }^{12}$ J. Young, J. Preston, H. van Driel, and J. Sipe, Phys. Rev. B 27, 1155 (1983).

${ }^{13}$ Y. Shimotsuma, K. Hirao, P. G. Kazansky, and J. Qiu, Jpn. J. Appl. Phys., Part 1 44, 4735 (2005).

${ }^{14}$ Y. Shimotsuma, K. Hirao, J. Qiu, and P. G. Kazansky, Mod. Phys. Lett. B 19, 225 (2005).

${ }^{15}$ D. Wortmann, J. Gottmann, N. Brandt, and H. Horn-Solle, Opt. Express 16, 1517 (2008).

${ }^{16}$ M. Lancry, B. Poumellec, A. Chahid-Erraji, M. Beresna, and P. G. Kazansky, Opt. Mater. Express 1, 711 (2011).

${ }^{17}$ Y. Shimotsuma, T. Asai, M. Sakakura, and K. Miura, J. Laser Micro/ Nanoeng. 9, 31 (2014).

${ }^{18}$ F. Zhang, H. Zhang, G. Don, and J. Qiu, J. Opt. Soc. Am. B 31, 860 (2014).

${ }^{19}$ T. Asai, Y. Shimotsuma, T. Kurita, A. Murata, S. Kubota, M. Sakakura, K. Miura, F. Brisset, B. Poumellec, and M. Lancry, J. Am. Ceram. Soc. 98, 1471 (2015)

${ }^{20}$ S. Richter, C. Miese, S. Döring, F. Zimmermann, M. J. Withford, A. Tünnermann, and S. Nolte, Opt. Mater. Express 3, 1161 (2013).

${ }^{21}$ S. Richter, D. Möncke, F. Zimmermann, E. I. Kamitsos, L. Wondraczek, A. Tünnermann, and S. Nolte, Opt. Mater. Express 5, 1834 (2015).

${ }^{22}$ F. Zimmermann, A. Plech, S. Richter, A. Tünnermann, and S. Nolte, Appl. Phys. Lett. 104, 211107 (2014).

${ }^{23}$ Technical Glasses, Physical and Technical Properties, SCHOTT North America, Inc. http://www.us.schott.com/tubing/english/download/schottbrochure-technical-glasses_us.pdf.

${ }^{24}$ K. Mishchik, A. Ferrer, A. Ruiz de la Cruz, A. Mermillod-Blondin, C. Mauclair, Y. Ouerdane, A. Boukenter, J. Solis, and R. Stoian, Opt. Mater. Express 3, 67 (2013).

${ }^{25}$ W. Yang, E. Bricchi, P. G. Kazansky, J. Bovatsek, and A. Y. Arai, Opt. Express 14, 10117 (2006).

${ }^{26} \mathrm{M}$. Gecevicius, M. Beresna, J. Zhang, W. Yang, H. Takebe, and P. G. Kazansky, Opt. Express 21, 3959 (2013).

${ }^{27}$ M. Shimizu, K. Miura, M. Sakakura, M. Nishi, Y. Shimotsuma, S. Kanehira, T. Nakaya, and K. Hirao, Appl. Phys. A 100, 1001 (2010).

${ }^{28}$ Y. Liu, M. Shimizu, B. Zhu, Y. Dai, B. Qian, J. Qiu, Y. Shimotsuma, K. Miura, and K. Hirao, Opt. Lett. 34, 136 (2009). 